\title{
Study on the techniques of wallpaper ("Biaohu") in the Palace Museum: Case on Lodge of Bamboo Fragrance(Zhu Xiangguan)
}

Ma Yue ( $D$ mayue_cc@hotmail.com )

Palace Museum

Congshan Zhao

Ministry of Culture and Tourism

Yong Lei

Palace Museum

Research article

Keywords: the Palace Museum, pattern, wallpaper

Posted Date: July 24th, 2021

DOI: https://doi.org/10.21203/rs.3.rs-724159/v1

License: (c) (i) This work is licensed under a Creative Commons Attribution 4.0 International License.

Read Full License 


\title{
Study on the techniques of wallpaper ("Biaohu") in the Palace
}

\section{Museum: Case on Lodge of Bamboo Fragrance(Zhu Xiangguan)}

\author{
MaYue $^{12 *}$, Zhao Congshan ${ }^{3}$, Lei Yong12
}

\begin{abstract}
Biao $\mathrm{Hu}^{\prime}$ " was one of the eight famous traditional crafts in the late Qing dynasty.Its function was decorating the interior of ancient buildings and making burial objects, including ceilings, walls and windows. It was popular in the buildings of northern China in the early Qing dynasty. There were white and patterned wallpaper in the Forbidden City, the latter included traditional and rare patterns in the Palace. Take the wallpaper in Lodge of Bamboo Fragrance(Zhuxiang Guan) as an example, in this article, its structure and composition are studied by morphological observation and spectral analysis. Combined with the analysis of the patterned wallpaper in other buildings of the Forbidden City, the traditional technology is studied.
\end{abstract}

Key words: the Palace Museum, pattern, wallpaper

\section{Background}

The traditional crafts in ancient China named "Ying Zao"." Biao Hu" was one of the eight crafts in late Qing dynasty. They were composed of tile, wood, stone, scaffold,soil,paint,colored painting and wallpaper.The use of paper and textile on the ceiling,wall and window was popular in the buildings of northern China in the early Qing dynasty. There were many records of it in the historical archives of Ming and Qing dynasties, which is related to the climate and building type in northern China. The wallpaper helps to prevent cold and dust, adjust the relative humidity, improve the illuminance,etc. There are a large number of wallpaper remains in the Forbidden City building, among which there are various decoration on it, most of which are green dragon with "厉" characters, as well as twigs and lotus, "prolonging

\footnotetext{
${ }^{*}$ Correspondence: mayue_cc@hotmail.com

1 Department of Conservation Science and Technology, The Palace Museum, 4 Jingshan Qianjie, Beijing 100009

2 Key Laboratory of Calligraphy and Paintings Conservation, Ministry of Culture and Tourism, Beijing, China

3 Department of Architectural Heritage, The Palace Museum, 4 Jingshan Qianjie, Beijing 100009
} 
life" patterns,etc. On the roof of the second floor of the south ear room in Zhu Xiangguan, rose flower decoration was found, which was rare in the Palace Museum.

Zhu Xiangguan is located in the northwest corner of the fourth courtyard in the Qianlong Garden, it was built in the $37^{\text {th }}$ year of Qianlong period of Qing dynasty, in imitation of the Bilin Pavilion of the Palace of Established Happiness (Jianfu Palace).It is divided into two floors, connected with Juan qinzhai and Yu cuixuan.The patterned wallpaper was found on the ceiling of the second floor during the conservation work, it has been taken off as a whole under conservation.
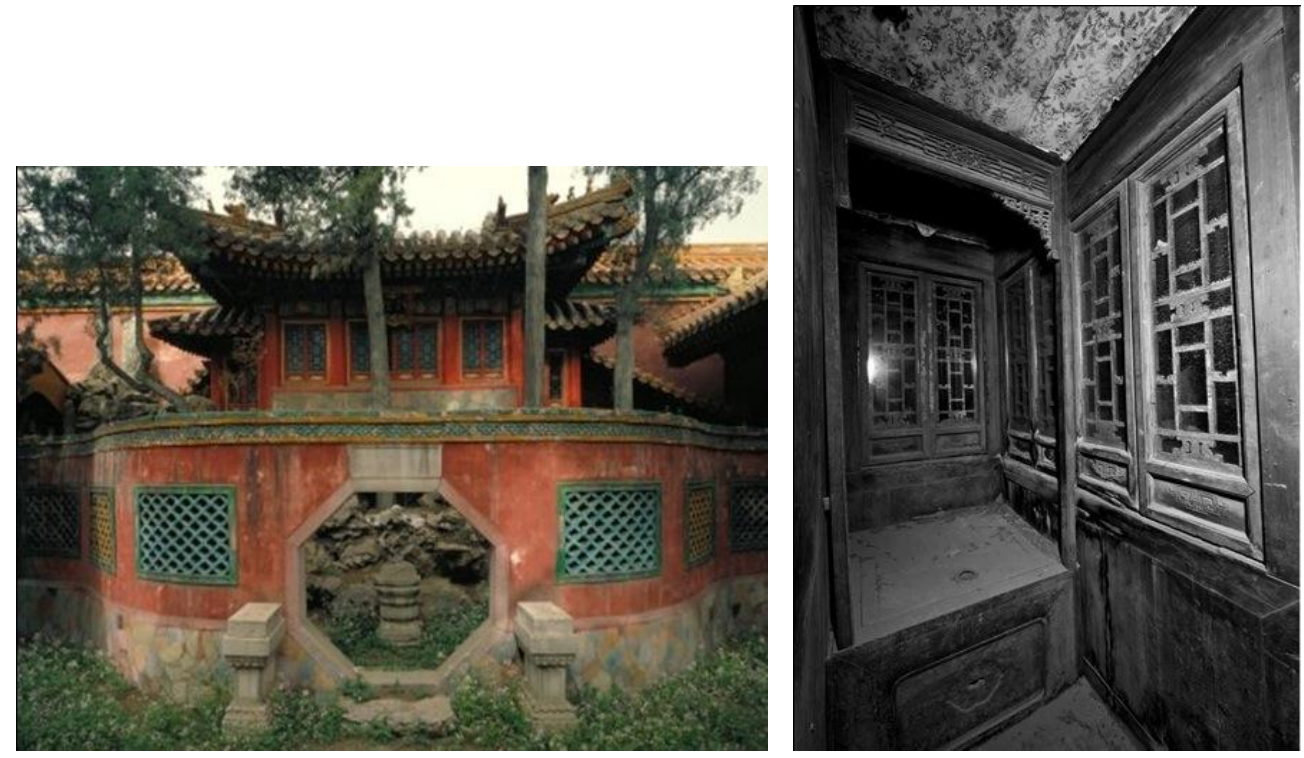

Fig.1 Exterior of Zhu xiangguan Fig.2 patterned wallpaper in Zhu xiangguan

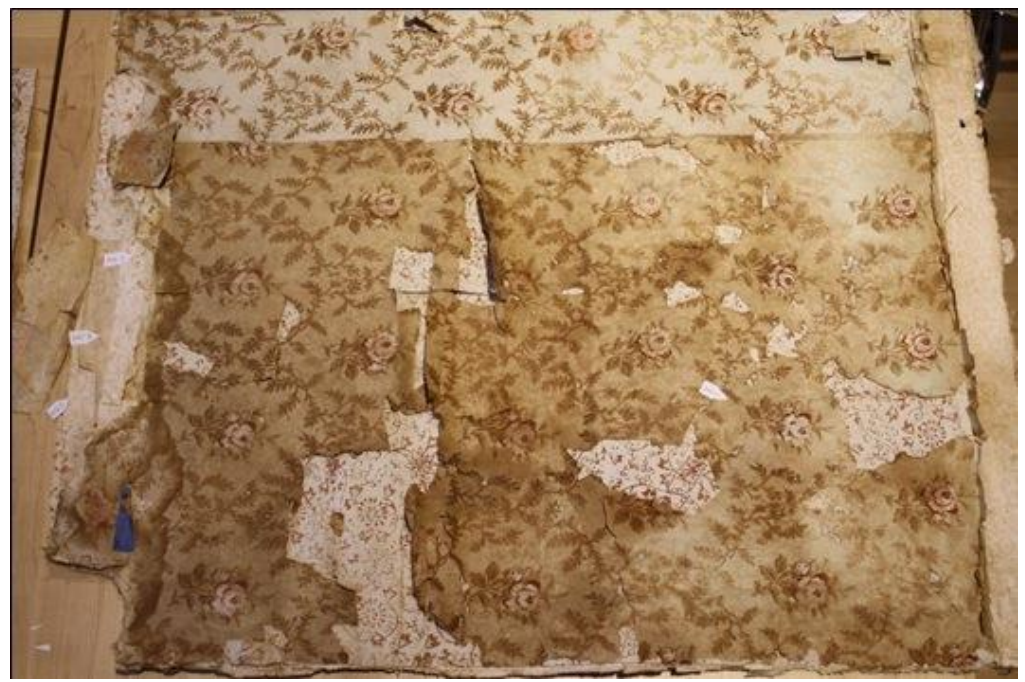

Fig.3 Sample of patterned wallpaper in Zhu xiangguan

The wallpaper appeared yellowing, cracking, missing and other diseases under environment. It can be divided into six layers, the pattern of the outermost layer is rose, the other layers are twigs. The structure and composition of the wallpaper were studied by microscopic observation and spectral analysis. 


\section{Scientific Analysis}

The sample is taken from edge of the wallpaper. It is embedded with Technovite 2000LC resin (Henraeus Kulzer Co.), polished after curing under ultraviolet light for 30 minutes. Observed under visible and ultraviolet light(Leica $4000 \mathrm{M})$, it can be divided to five layers at least.

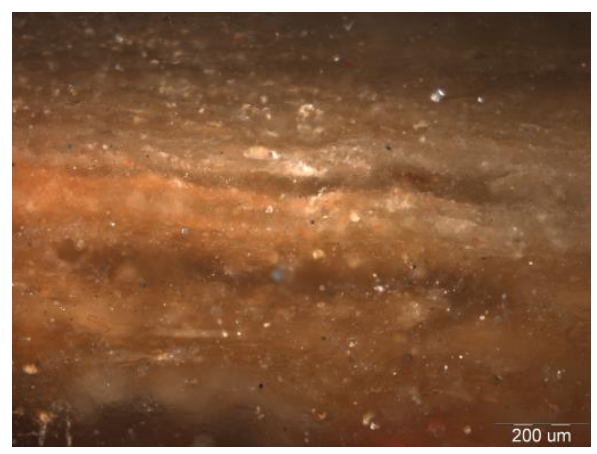

Fig.4 Microstructure of crosssection

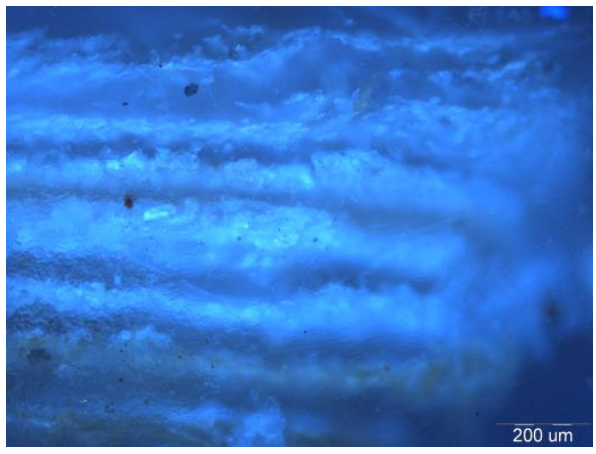

Fig.5 Microstructure of crosssection

UV, $100 \mathrm{X}$

The structure of pigment and fiber were observed by microscope. As an example, on the surface of the fifth layer (the outermost layer) and the second layer, the pigment and paper fiber are different, so component analysis is required.

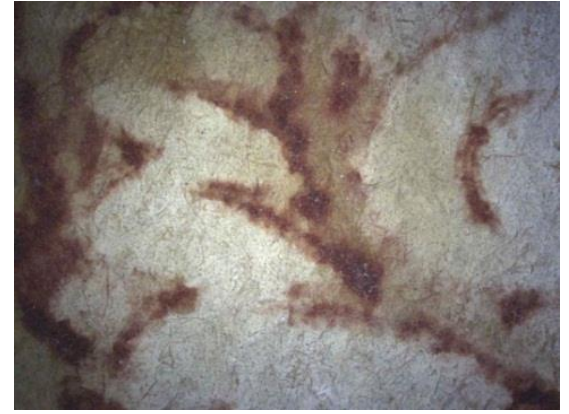

Fig.6 Microscopic morphology of the $5^{\text {th }}$ layer $20 \mathrm{X}$

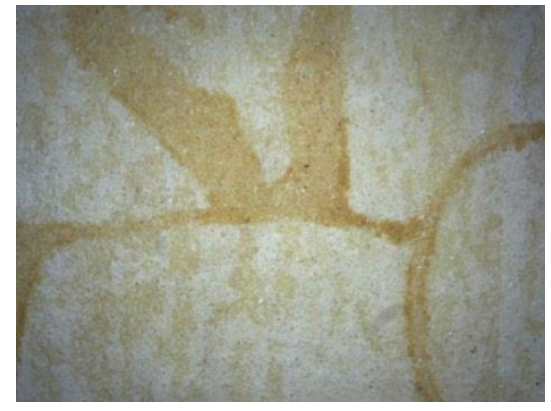

Fig. 8 Microscopic morphology of the 2nd layer $20 \mathrm{X}$

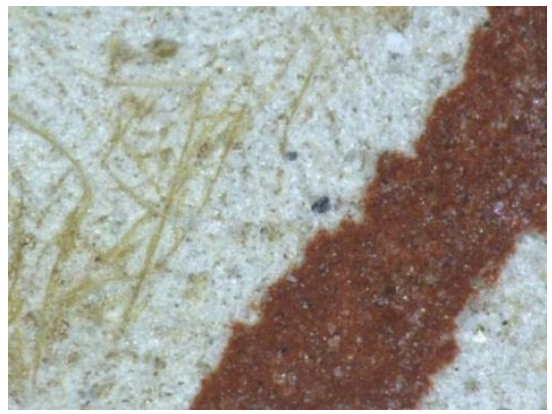

Fig.7 Microscopic morphology of the $5^{\text {th }}$ layer $150 \mathrm{X}$

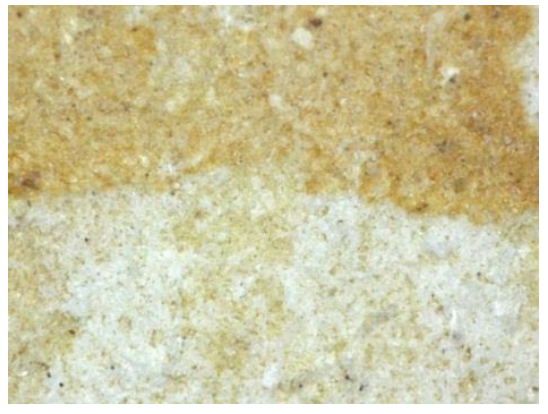

Fig.9 Microscopic morphology of the 2nd layer $150 \mathrm{X}$ 
Micro-XRF imaging analysis was taken on surface of the patterned wallpaper. From the distribution of element, it shows that the red vein lines of flowers mainly contain $\mathrm{Ba}$, brown yellow leaves and mainly contain $\mathrm{Cr}$. The white part of the flower is rich in Ca.The distribution of Fe reflects the red pattern on the surface of layer1-4.The specific components of each part of the material need to be further analyzed.

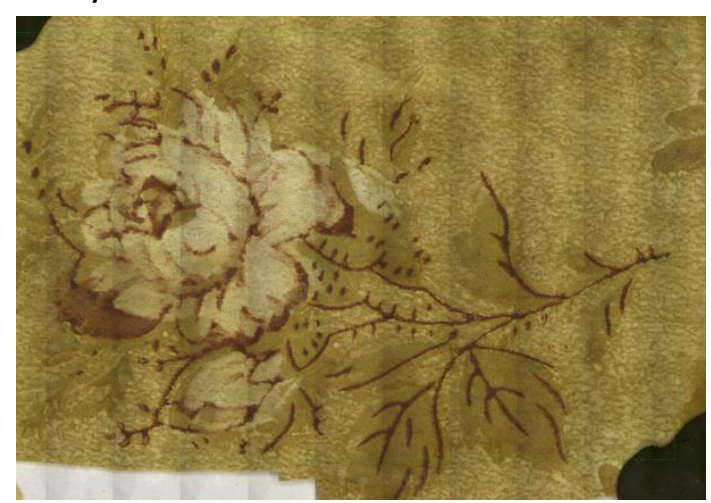

Fig. 10 the $5^{\text {th }}$ layer of patterned wallpaper

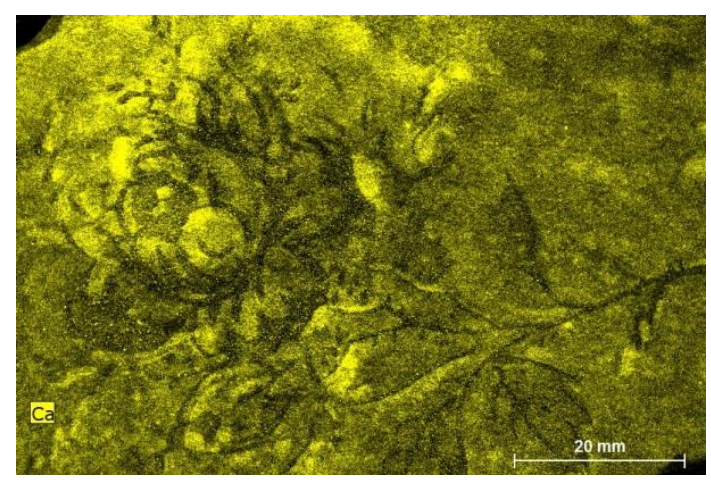

Fig.12 Mapping of $\mathrm{Ca}$

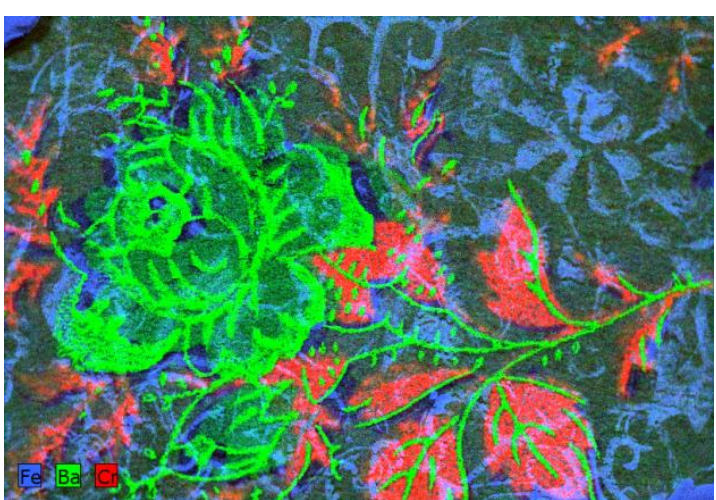

Fig.11 Mapping of $\mathrm{Fe} / \mathrm{Ba} / \mathrm{Cr}$

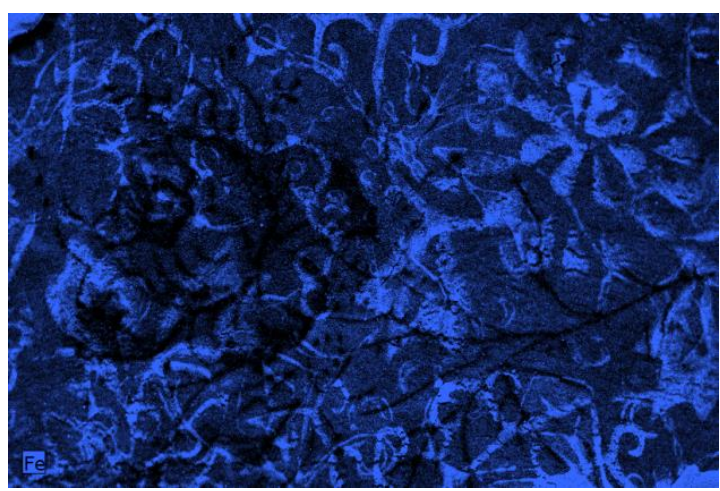

Fig.13 Mapping of Fe

The distribution of elements of the fourth layer was analyzed,Fe is mainly existed in the red pigment.

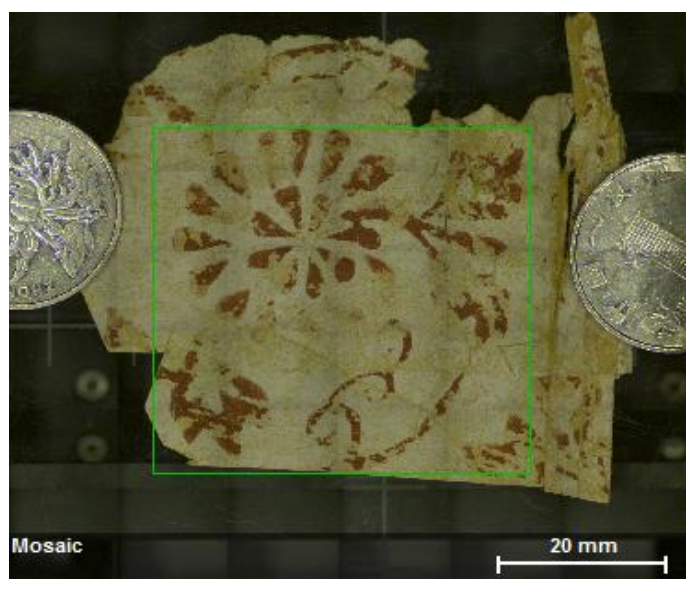

Fig. 14 the $4^{\text {th }}$ layer of patterned wallpaper

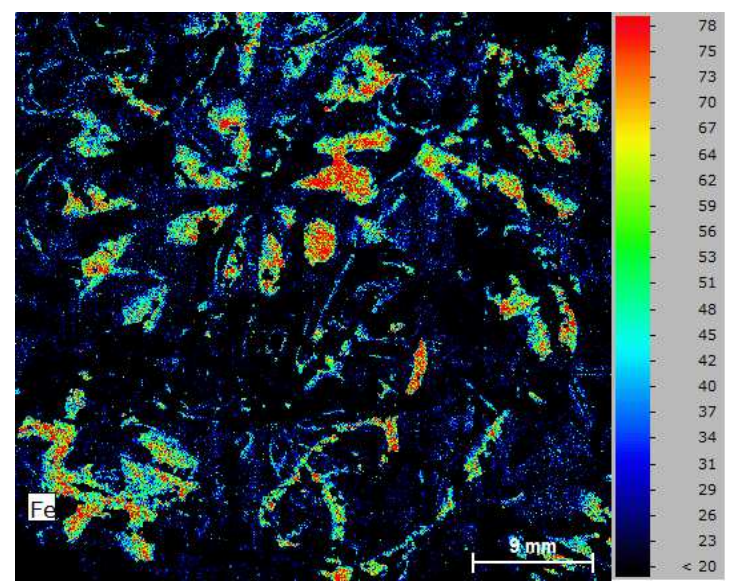

Fig.15 Mapping of $\mathrm{Fe}$

Red and brown sample from the fifth layer were observed by polarized microscope (Leica,4500P). Organic dyes may be used in the red vein. A small amount 
of red sample were taken by ultra-high performance liquid chromatography-time-of-flight mass spectrometry (Waters UPLC H-class) and Waters G2-XS QTOF MS. After $10 \mathrm{~min}$, was extracted by ultrasound at $70{ }^{\circ} \mathrm{C}$ with $0.1 \mathrm{~mL}$ DMSO, centrifuge, remove the clear liquid and set aside, the residue in $0.1 \mathrm{~mL}$ $\mathrm{MeOH} / \mathrm{H} 2 \mathrm{O} / \mathrm{HCl}(1: 1, \mathrm{~V} \leq \mathrm{V})$ at $70{ }^{\circ} \mathrm{C}$. was extracted by ultrasonic at $70{ }^{\circ} \mathrm{C}$ for 10 min, and then concentrated and redissolved with clear liquid of first step, then to be measured

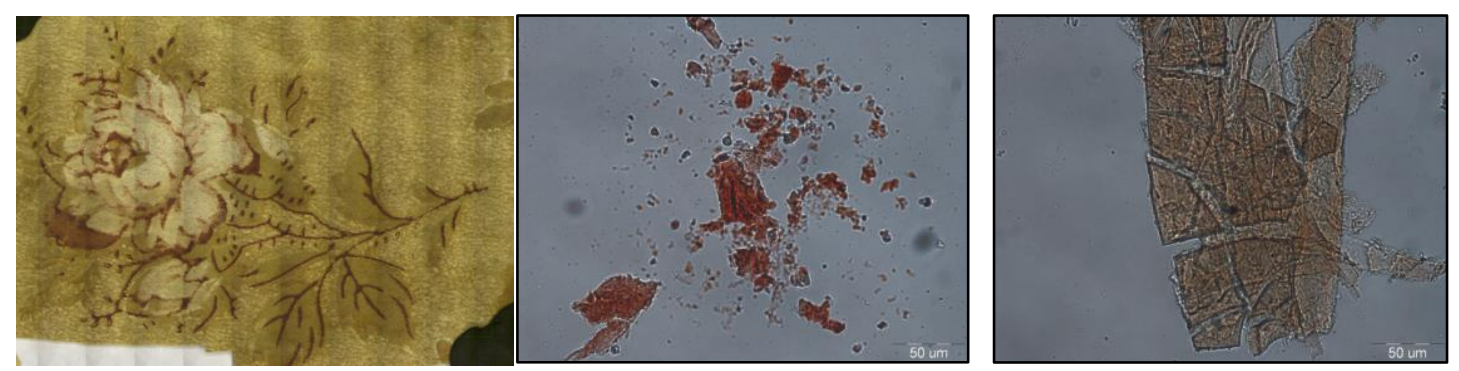

Fig.16 Microscopic morphology of Fig.17 Microscopic morphology of red dye(Single polarization) brown dye(Single polarization)

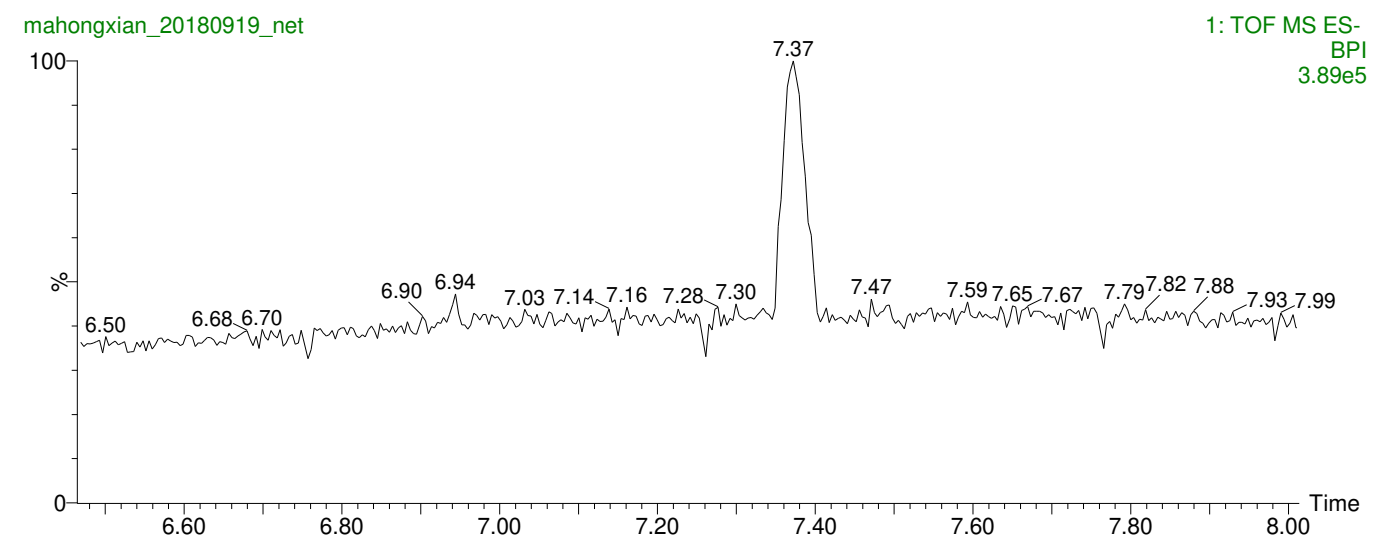

Fig.18 Chromatogram of the red dye(UPLC-QTOF-MS ESI)

mahongxian_20180919_net 1733 (7.372)

100

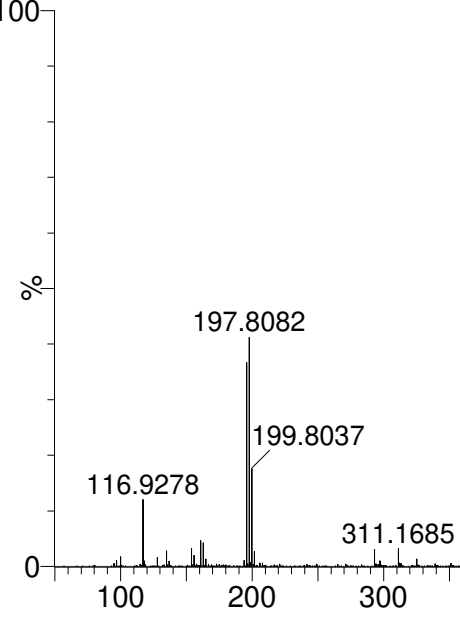

646.7031

644.7044

648.6995

642.7038650 .6990

Fig.19 Mass spectrum at $37 \mathrm{~min}$ 
Table 1 Compound analysis

\begin{tabular}{|c|c|c|c|}
\hline 样品编号 & 保留时间 $/ \min$ & 母离子 $m / z(-)$ & 化合物 \\
\hline 红色勾线样品 & 7.37 & 646.703 & 酸性红 87 (曙红) \\
\hline
\end{tabular}

According to the analysis by Ultra High Performance Liquid

Chromatography-Mass Spectrometry, the red vein may have used a synthetic dye, which is relatively rare on the wallpaper in the Palace Museum, which may be of the later communication or renovation between China and the West. This requires further discussion.

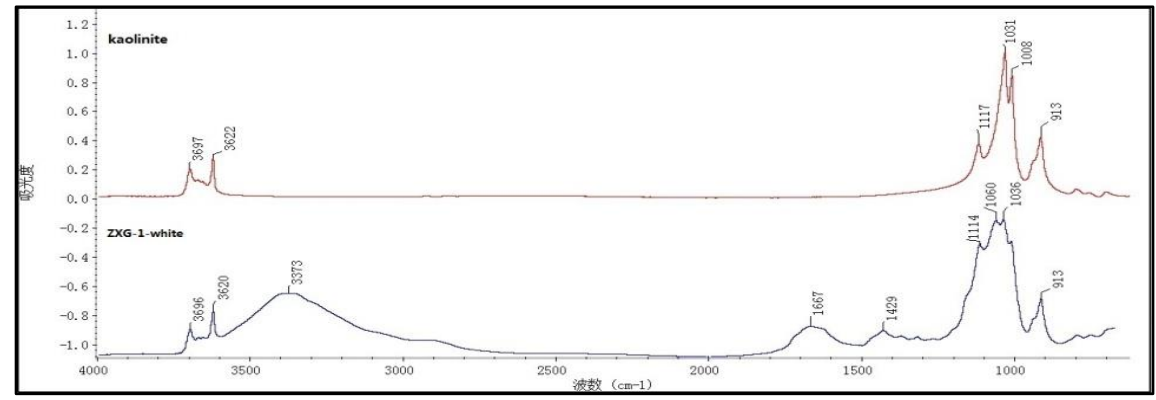

Fig.20 Micro-FTIR spectrum of white pigment the $5^{\text {th }}$ layer

The main component of the white part of the flower is the kaolinite group mineral and the lime.

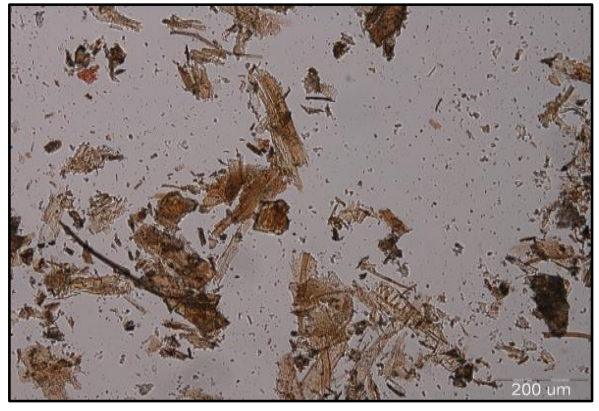

Fig.21 Microscopic morphology of the $5^{\text {th }}$ layer (Single polarization)

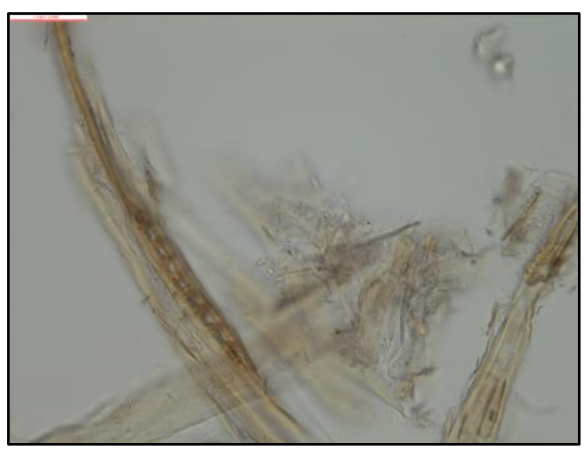

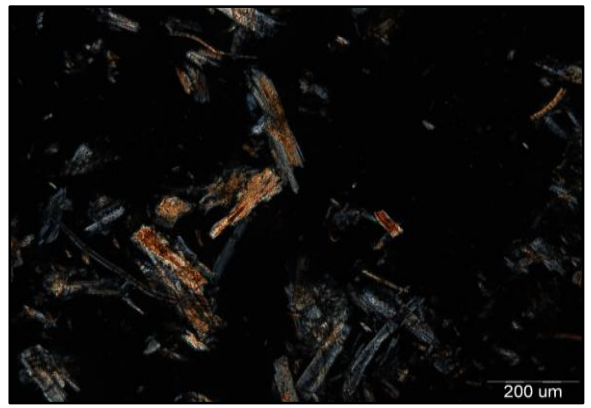

Fig.22 Microscopic morphology of the $5^{\text {th }}$ layer (Orthogonal polarization)

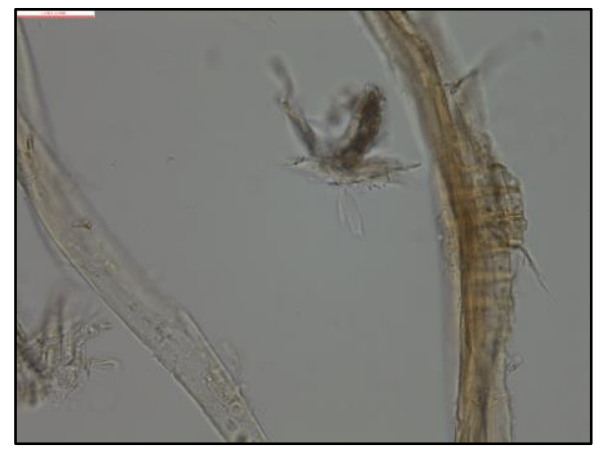

Fig.23 Microscopic morphology of the $5^{\text {th }}$ layer Fig.24 Microscopic morphology of the $5^{\text {th }}$ layer (transmitted light, pit of wood cell) (transmitted light,wood ray) 
Paper fiber of the fifth layer was observed under polarized and transmission microscope.The shape of sawdust under polarized light,the hole and wood ray structure under transmission light all proved it to be wood fiber. Maybe this layer was pasted later from the 1-4 layers.

Decoration on the 1-4 layers are traditional types in Qing dynasty. From the red pigment and microstructure of paper fiber analysis, the red pigment may be iron red, and the white bottom ash contains talc, the paper mainly contains bamboo fiber. It is similar as other buildings studied by the author. It all shows that traditional materials and technique were used in 1-4 layers. Because the surface of the third layer is seriously wore, and the pigments on the 1-3 layer is more orange, so it is speculated that the1-3 layers were pasted together, and the fourth layer was pasted later.So the 1-6 layers of wallpaper has been pasted at least 3 times.

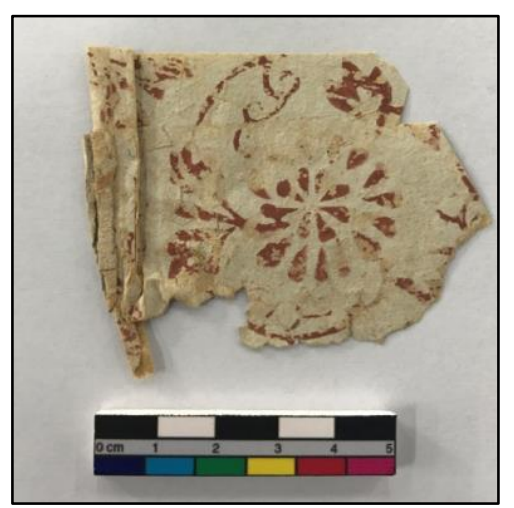

Fig. 25 the $4^{\text {th }}$ layer of patterned wallpaper

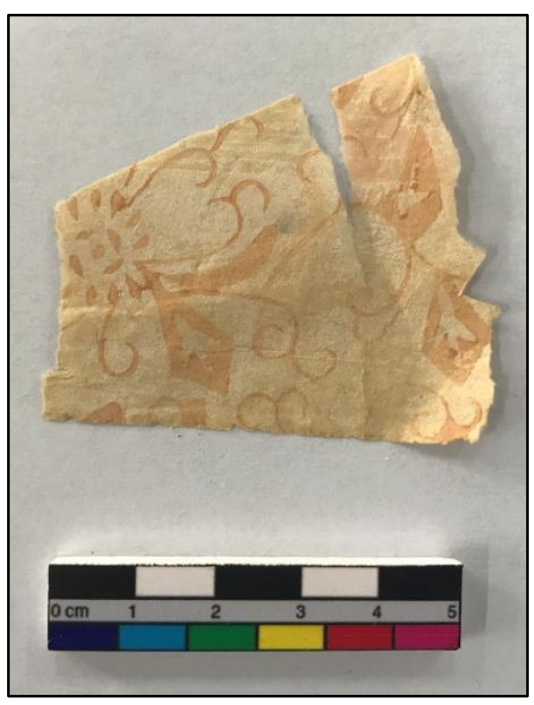

Fig.27 the $2^{\text {nd }}$ layer of patterned wallpaper

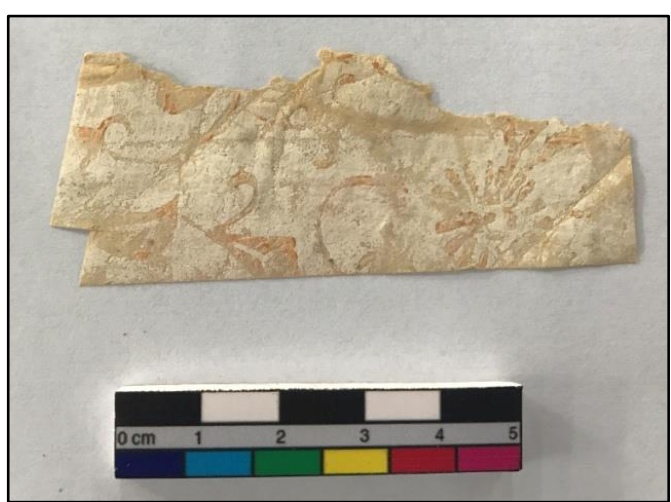

Fig. 26 the $3^{\text {th }}$ layer of patterned wallpaper

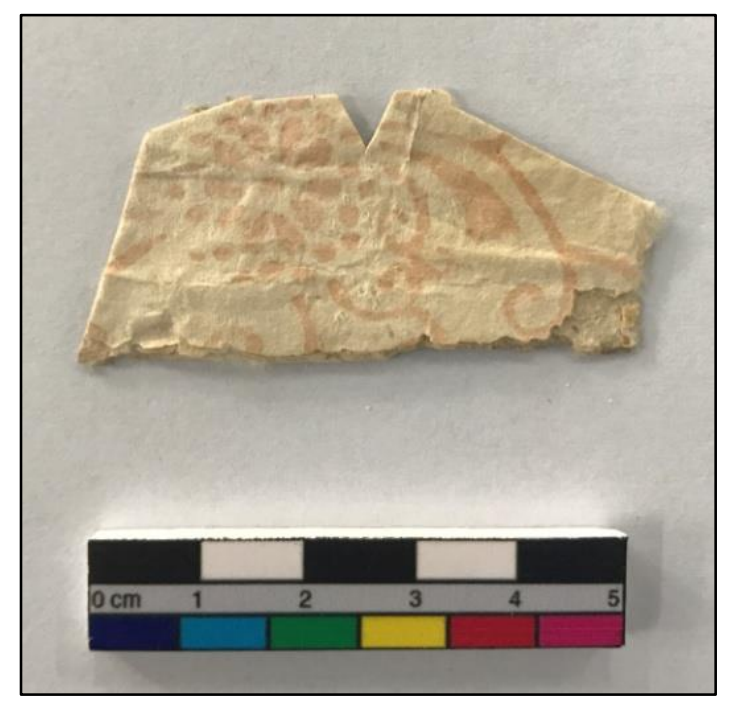

Fig. 28 the $1^{\text {th }}$ layer of patterned wallpaper 


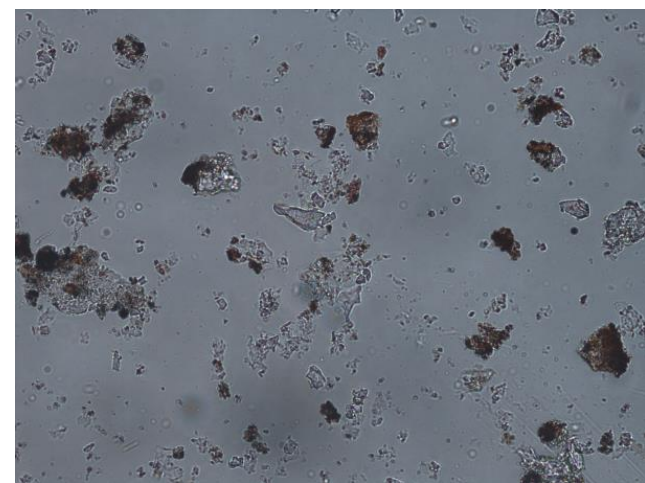

Fig.29 Microscopic morphology of the red dye on the $4^{\text {th }}$ layer

(Single polarization)

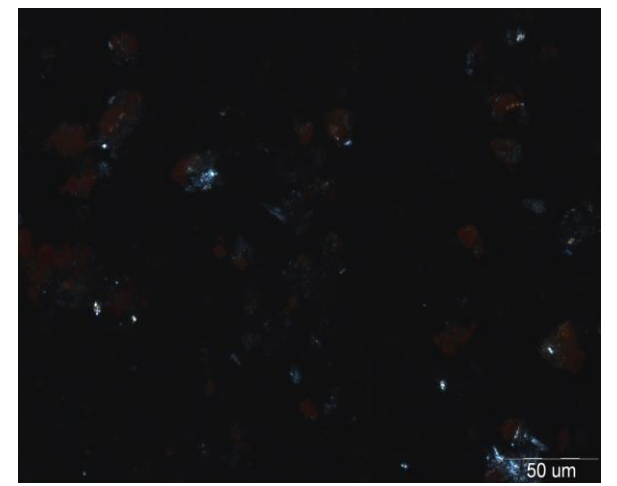

Fig.30 Microscopic morphology of the red dye on the $4^{\text {th }}$ layer

(Orthogonal polarization)

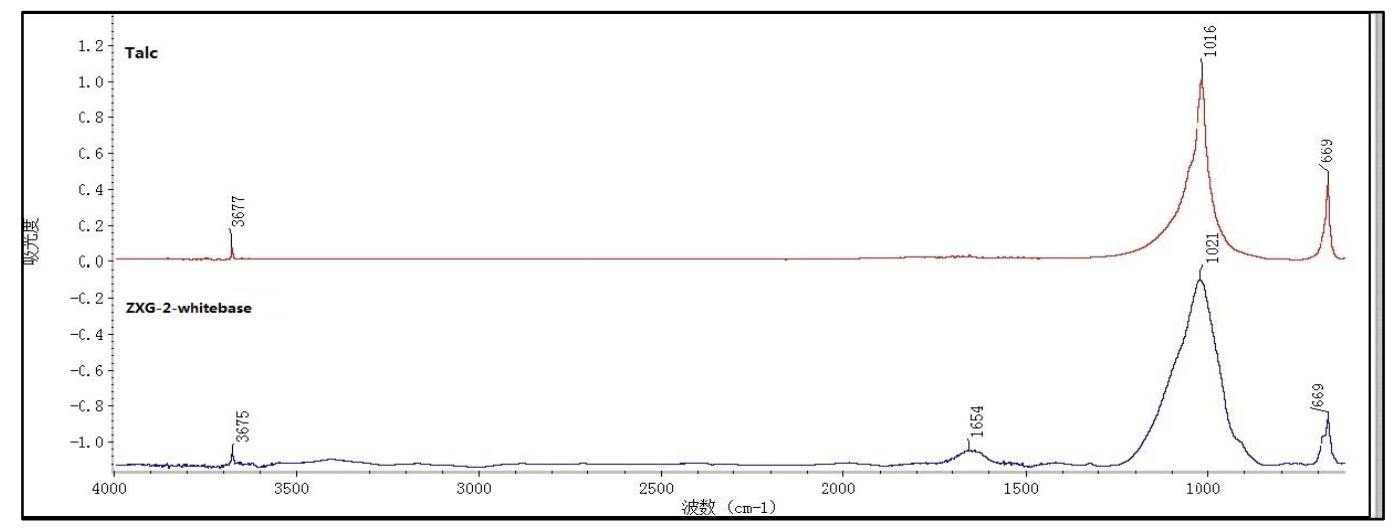

Fig.31 Micro-FTIR spectrum of white pigment the $4^{\text {th }}$ layer

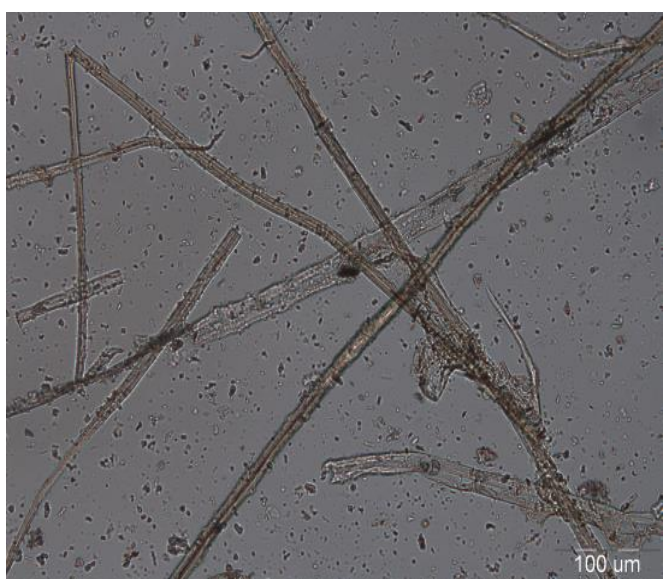

Fig.32 Microscopic morphology of the $4^{\text {th }}$ layer (Single polarization)

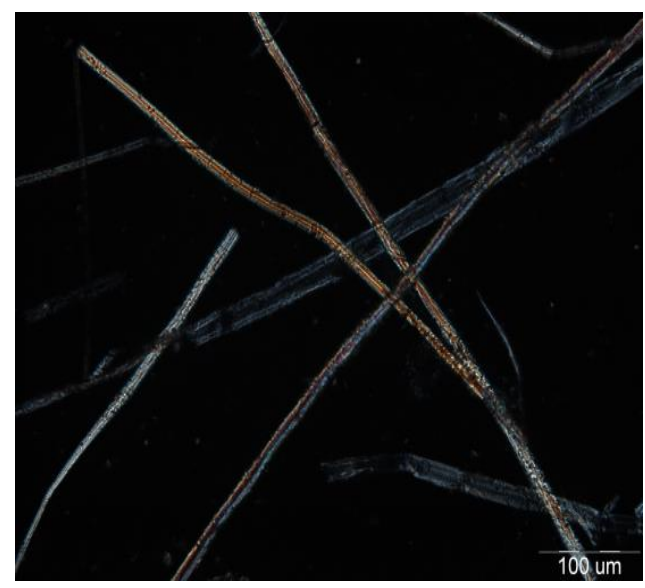

Fig.33 Microscopic morphology of the $4^{\text {th }}$ layer (Orthogonal polarization) 


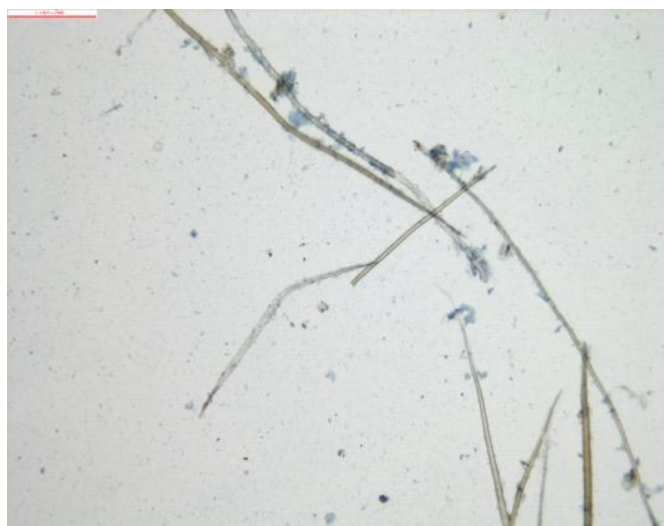

Fig.34 Microscopic morphology of the $4^{\text {th }}$ layer Fig. 35 (transmitted light, pit of wood cell)

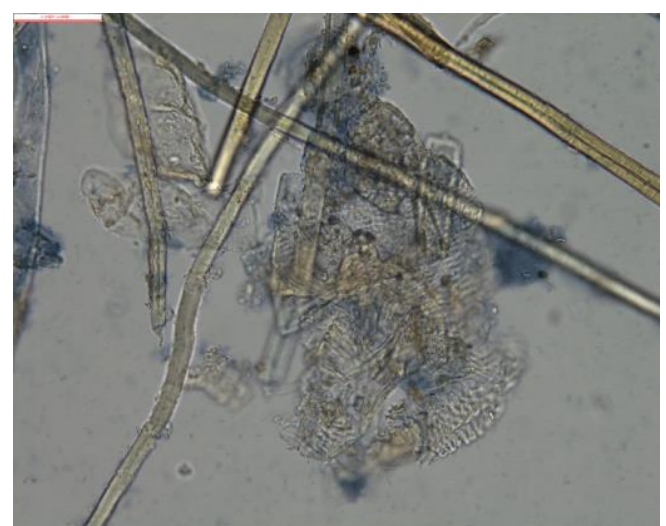

Microscopic morphology of the $4^{\text {th }}$ layer (transmitted light, wood vessel)

\section{Wallpaper in in the Hall of Mental Cultivation(Yangxin Dian)}

During investigation of the wallpaper in Yangxin Dian, many types of patterned wallpaper was found. The following are examples compared with it in Zhu Xiangguan, traditional decoration and the one using synthetic pigment are discussed.

\section{1 Wallpaper with dragon and “尼” patterned decoration}

The residue of patterned wallpaper was found on the backing wall in the Hall of Following the Practice(Tishun tang), it is decorated with traditional dragon and "Zt". The distribution of surface elements, green pigment and paper fiber are analyzed.The results show that atacamite, lime,talc for white base and bamboo paper were used. They are all typical traditional materials in the Qing dynasty.
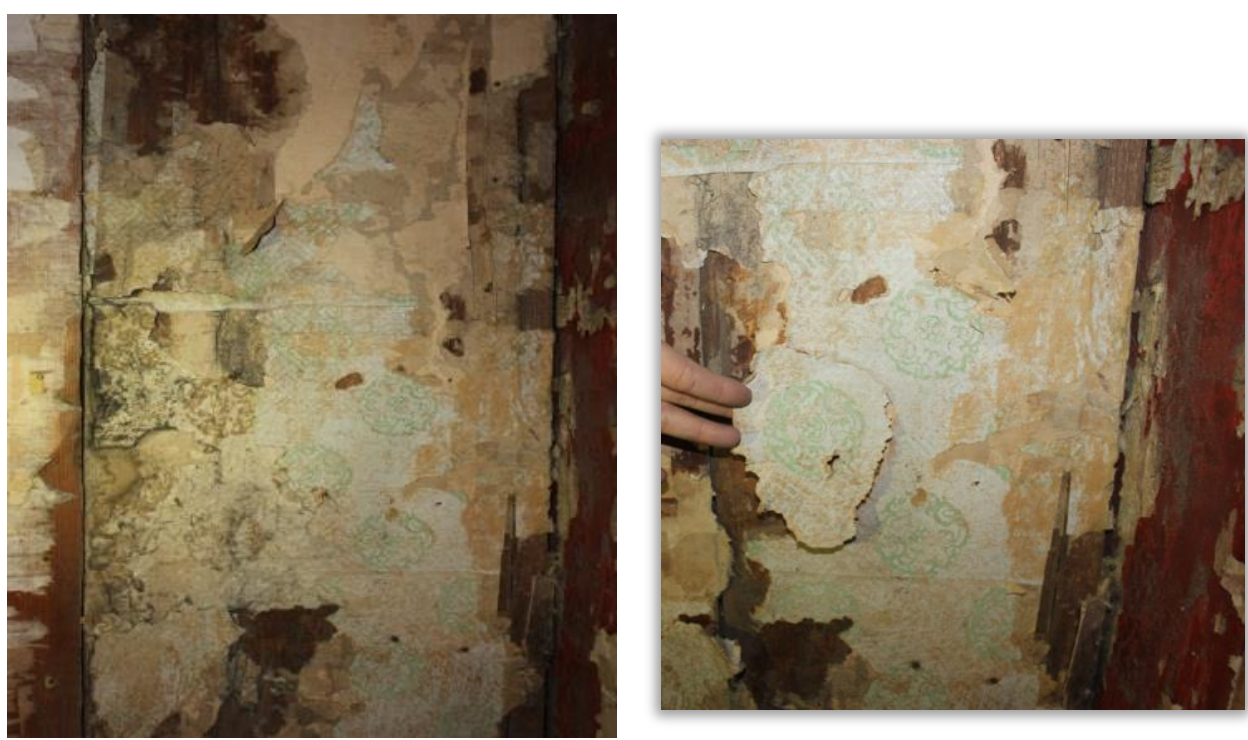

Fig.36 patterned wallpaper on the west room of Ti shuntang in Hall of Mental Cultivation (Yangxin dian) 


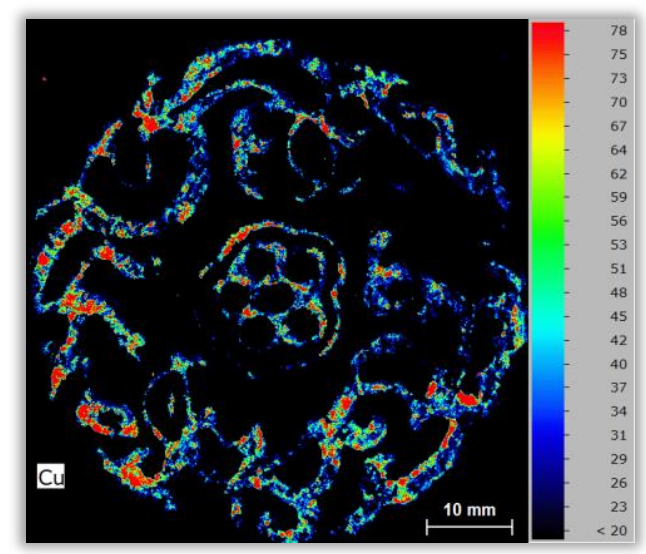

Fig.37 Mapping of $\mathrm{Cu}$

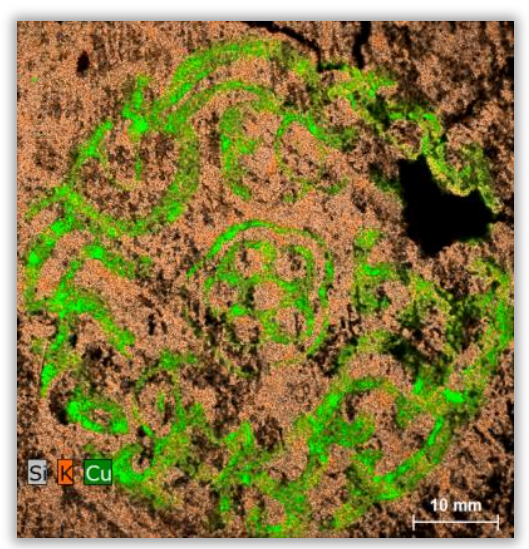

Fig.38 Mapping of $\mathrm{Cu} / \mathrm{Si} / \mathrm{K}$

3. 2 Patterned wallpaper on the wall surrounding the bed(Kang) in the western buildings of Yangxin Dian

The wallpaper on the wall surrounding the bed(Kang)is quite different from others, the pigment and technique were analyzed.

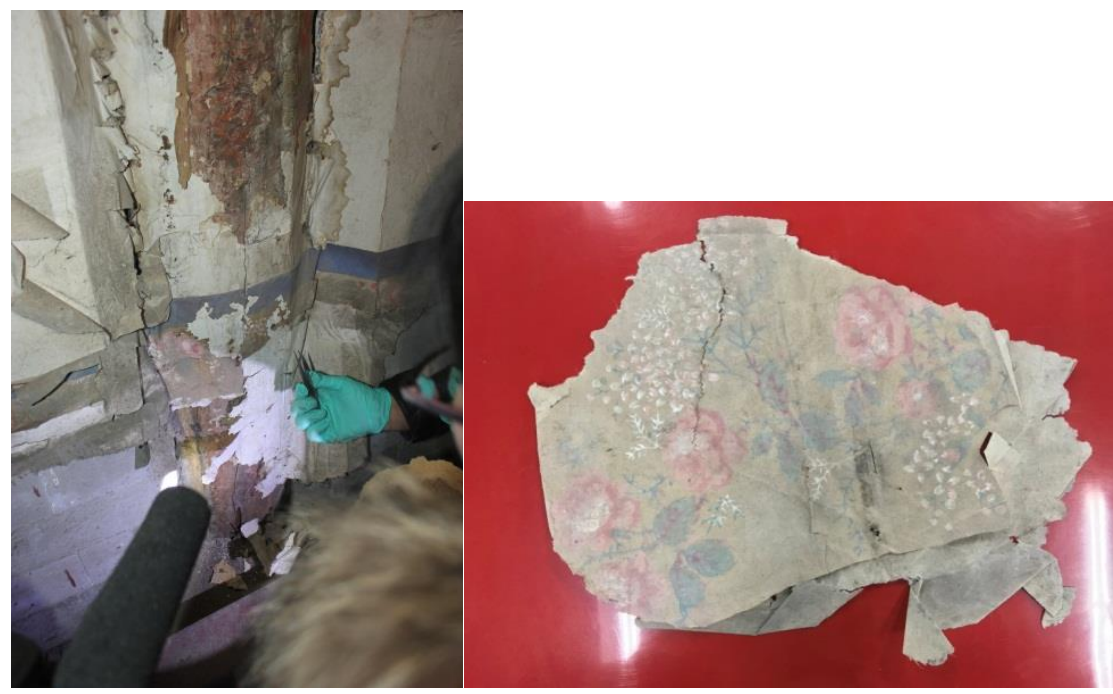

Fig.39 patterned wallpaper on surrounding of the bed in in Hall of Mental Cultivation (Yangxin dian)

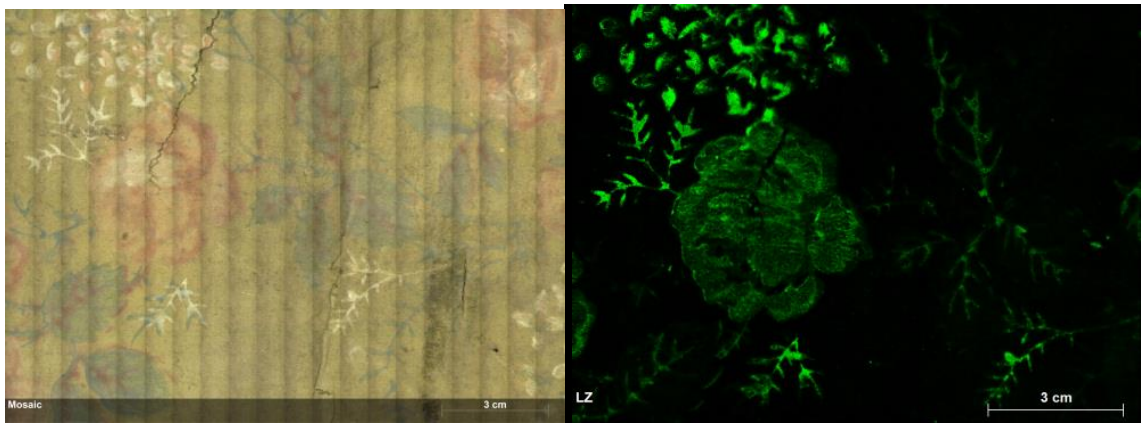

Fig.40 Mapping of Ba 


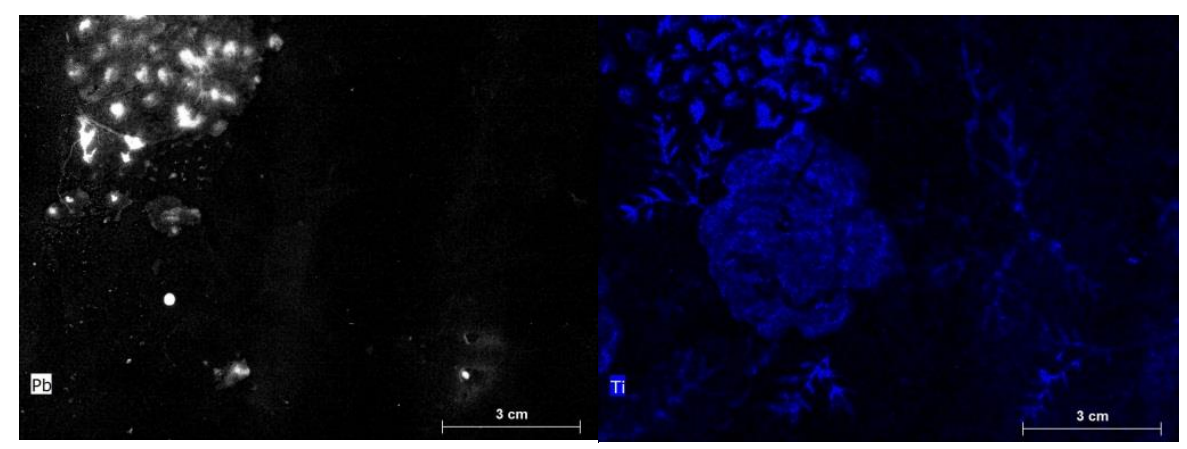

Fig.41 Mapping of $\mathrm{Pb}$

Fig.42 Mapping of $\mathrm{Ti}$

The white pigments includes $\mathrm{Pb}, \mathrm{Ba}$ and $\mathrm{Ti}$, so titanium white and barium white. It also proves that this wallpaper was pasted later, and the time can be further discussed with the dye analysis.

\section{Discussion}

In the study of wallpaper in the Palace Museum, there are many kinds of patterned ones, but it most common in Qing Dynasty are traditional decoration, such as green dragon, twigs and lotus, curly grass and so on. However, in the investigation of several patterned wallpaper in Zhuxiang Guan and Yangxin Dian, we found that some of the outermost layer used modern synthetic dye or pigment, and the paper was also with wood pulp. The surface of these wallpaper were richly decorated, which is different from the traditional style of Qing Dynasty. According to the comparison with western wallpaper, they are similar in decoration. These wallpaper may be related to the communication between China and the West, and the times and techniques between them will be studued in conjunction with the literature in the future.

Acknowledgements: Thanks are given to Duan Pei-quan helped me perform testing with XRF.This research could not have been completed without his help.

Authors' contributions: YM performed most of the analytical work and was a major contributor in writing the manuscript. CSZ provided us the sample to study it. YL introduced crafts of the wallpaper. All authors read and approved the final manuscript.

Funding :This research was supported by the National Key R\&D Program of China, No. 2019 YFC1520300.

Availability of data and materials The datasets used and/or analyzed during the current study are available from the corresponding author upon reasonable request. 
Declarations Competing interests The authors declare that they have no competing interests.

\section{Author details}

1 Department of Conservation Science and Technology, The Palace Museum, 4 Jingshan Qianjie, Beijing 100009, China.

2 Key Laboratory of Calligraphy and Paintings Conservation, Ministry of Culture and Tourism, Beijing, China

\section{References:}

1.Yang Hong, 2004.The traditional craft"Biao Hu" in the Forbidden City.Forbidden City,6:88-97. 2.Wang Zhongjie,1995. "Biao Hu” in the Qing dynasty. Forbidden City,1:45.

3.Zhang Yaping,2015. The preliminary study of Lodge of Bamboo Fragrance(Zhuxiang Guan).Beijing Cultural Relics and Museums,2:59-65. 